\title{
The contribution of PCR in the diagnosis of postnatal pulmonary infections
}

\author{
F. Bennaoui ${ }^{1,2}$, S.Mrhar ${ }^{1,2}$, M.Naaim ${ }^{1,2}$ N. El Idrissi Slitine ${ }^{1,2}$, N.Soraa ${ }^{3}$, F.M.R Maoulainine \\ 1-Neonatal Intensive Care Department, Mohammed VI University Hospital and Research \\ 2-Team for Childhood, Health and Development, Marrakech School of Medicine, Cadi Ayyad University, \\ Marrakech, Morocco \\ 3- Microbiology Virologyy Departement, Mohammed VI University Hospital and Research
}

\section{INTRODUCTION}

Postnatal pulmonary infection remains a serious cause of mortality and neonatal morbidity. Its etiological diagnosis is not often obvious and the use of PCR was not systematic.

The aim of this work is to demonstrate the interest of PCR in the diagnosis of postnatal pulmonary infections.

\section{PATIENTS AND METHODS}

- Comparative retrospective study

- Neonatal intensive care unit, MOHAMMED VI University Hospital ;Marrakech.

- Sixty-six patients aged 7 to 40 days, with postnatal respiratory distress.

- Two periods of study: the first between January 2014 and March 30, 2015 (population A), and the second from January 1, 2018 to April 30, 2018 (population B).

\section{RESULTS}

- Thirty girls and thirty-six boys; sex ratio (0.45)

- Age of admission (10 to 40 days with an average age of 18 days)

- Infectious contagion was found in $34 \%$ of cases.

- Respiratory distress was present in all newborns evaluated by Silverman score (Fig.1)

- Population A did not benefit from a PCR examination, except population B made of 20 patients all benefited from a PCR positive income test, making a percentage of $30.3 \%$ of the total population.

- PCR isolated viruses in all cases and mycoplasma in one case (Fig.2)

- Population A was treated with 3rd generation cephalosporins and gentamicin associated with macrolides in 34\%. Population B was treated with macrolides alone in $10 \%$ and just with respiratory physiotherapy,

- Mean hospital stay was 10 days in population A, and 5 days in population $\mathrm{B}$.

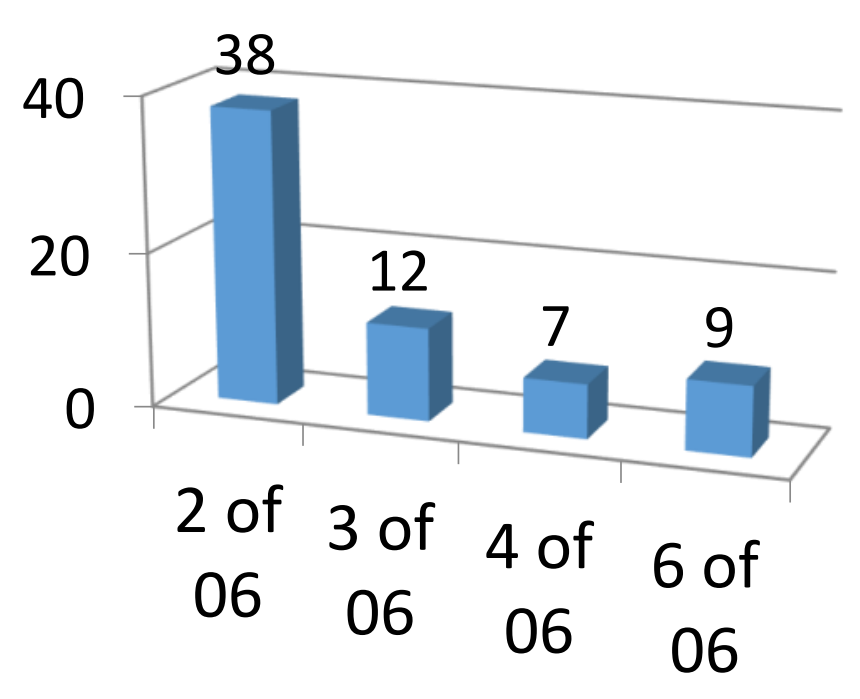

Figure 1 : Distribution of respiratory distress according to Silverman score

2

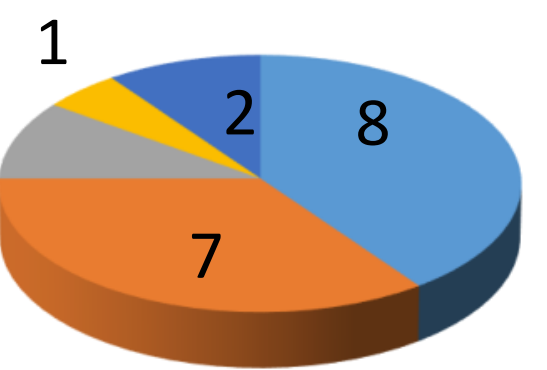

rhinovirus

VRS+adenovi rus

$\square$ VRS+ mycoplasme

VRS + rhinovirus

Figure 2: Germs isolated by $P C R$

\section{DISCUSSION}

- Postnatal pulmonary infections or late-onset lung infections are defined by pulmonary parenchymal infection during the neonatal period and after a latency period exceeding 3 days of life

- The clinical picture is dominated by the syndrome of respiratory distress of the newborn, causing first suspicion of pneumonitis of infectious origin, whose germs are multiple dominated by viral, bacterial and more rarely fungal agents.

-Advances in diagnostics include the use of new radiological methods, better blood and microbiological samples, and better microbiological tests.

-Real-time PCR is usually the most sensitive and fastest method giving results in less than 24 hours.

- The negative predictive value of these tests is generally excellent and can (if these results are rapid) reduce the unnecessary consumption of empirically prescribed antibiotics.

- When no germ is documented by conventional molecular and culture approaches, broad-spectrum eubacterial PCR can be performed on normally sterile specimens such as pleural fluid or lung biopsies.

\section{CONCLUSION}

Significant changes in management modalities have been observed with the use of PCR in the diagnosis of postnatal pulmonary infection. 